\title{
Inhaled amikacin for severe Gram-negative pulmonary infections in the intensive care unit: current status and future prospects
}

\author{
Antoni Torres ${ }^{1,2,3,4^{*}}$, Anna Motos ${ }^{1}$, Denise Battaglini ${ }^{1,5}$ and Gianluigi Li Bassi ${ }^{1,2,3,4}$
}

\begin{abstract}
Recently, the use of nebulized antibiotics in the intensive care unit, in particular amikacin, has been the subject of much discussion, owing to unconvincing results from the latest randomized clinical trials. Here, we examine and reappraise the evidence in favor and against this therapeutic strategy; we then discuss the potential factors that might have played a role in the negative findings of recent clinical trials. Also, we call attention to several factors that are seldom considered by study developers and regulatory agencies, to promote translational research in this field and improve the design of future randomized clinical trials.
\end{abstract}

Keywords: Pseudomonas aeruginosa, Amikacin, Ventilator-associated pneumonia, Gram-negative bacteria

In light of the latest negative results from large randomized clinical trials, i.e., the IASIS [1] and the INHALE II trials [2], evaluating the efficacy of inhaled amikacin in critically ill mechanically ventilated patients, scientists are calling into question the merits of the use of inhaled antibiotics in the field of critical care medicine. In this review we elaborate on some of the most cogent reasons for continuing investigating the merits of inhaled antibiotics, and we extrapolate from the latest undesirable findings. Furthermore, we provide our perspective on the next potential targets, in both clinical and laboratory research.

\footnotetext{
*Correspondence: atorres@clinic.ub.es; atorres@clinic.cat; http://www. idibapsrespiratoryresearch.org

${ }^{1}$ Department of Pulmonary and Critical Care Medicine, Hospital Clinic, Calle Villarroel 170, Barcelona 08036, Spain

${ }^{2}$ Institut d'Investigacions Biomèdiques August Pi i Sunyer (IDIBAPS),

Barcelona, Spain
}

Full list of author information is available at the end of the article

\section{The therapeutic promise of inhaled amikacin}

Severe respiratory infections developed in hospital settings, i.e., hospital-acquired pneumonia (HAP) and ventilatorassociated pneumonia (VAP), are common [3] and constitute a significant burden for healthcare systems. VAP, which is commonly caused by aerobic Gram-negative bacilli [6], prolongs hospitalization [4] and consequently increases hospital costs [5].

In hospital settings, multi-drug resistant (MDR) pathogens are prevalent, which makes choosing the most appropriate empiric therapy extremely challenging. The latest American [3] and European guidelines [7] for the management of HAP/VAP patients provided recommendations to tackle this problem. Yet, according to their suggestions, in settings with high levels of antibiotic resistance, the use of narrow-spectrum empiric antibiotics is impractical, creating a vicious cycle that could even sustain MDR. Furthermore, applicability of clinical practice guidelines is uncertain in countries defined by the highest prevalence of pandrug resistant (PDR) pathogens and widespread use of antibiotics. As a result, in upcoming years, nosocomial MDR is expected to rise globally.

Bearing in mind this threatening scenario, the inherent limitations of intravenous antibiotics for the treatment of pulmonary infections should be emphasized. Indeed, intravenous antibiotics often present insufficient pulmonary distribution [8]. Laboratory evidence has also consistently confirmed marginal concentrations of antibiotics in bacterial biofilm retained into endotracheal tubes [9]. Additionally, intravenous antibiotics are often underdosed in patients with a sepsis-related hyper-dynamic state or larger distribution volumes for vast edema [10]. These factors intensify the selective pressure for the development and worsening of MDR. In theory, administration of nebulized antibiotics is a potential therapeutic alternative [11] to overcome these limitations. Nebulized antibiotics could deliver an effective amount of the drugs directly into 
the respiratory system, overcoming minimal inhibitory concentrations (MICs), while thwarting selective pressure and MDR development [12]. Additionally, systemic exposure to antibiotics and adverse effects could be reduced. Among the available antibiotics that could be nebulized into the respiratory system, aminoglycosides have drawn much attention, since they are concentration-dependent antibiotics with post-antibiotic effect and present a broad spectrum of activity. Aminoglycosides have been already applied as chronic therapies for cystic fibrosis patients with difficultto-treat infections [13]. In critically ill patients, nebulized amikacin could attain substantial pulmonary concentrations, and achieve outstanding bactericidal efficacy. Moreover, nephrotoxicity and ototoxicity, which are commonly associated with intravenous administration of amikacin, could be curtailed.

A number of laboratory and clinical studies have corroborated the merits of inhaled amikacin in VAP. In an animal study, Goldstein and collaborators [14] administered $45 \mathrm{mg} / \mathrm{kg}$ nebulized amikacin in pigs with Escheichia coli severe pneumonia. Amikacin concentration in the most severely infected pulmonary regions averaged $40 \pm$ $65 \mu \mathrm{g} / \mathrm{g}$, achieving tissue concentrations 30 times higher than intravenous amikacin. In a pivotal study by Lu et al. [15], 40 patients with Pseudomonas aeruginosa VAP were included in a randomized study. Twenty patients with susceptible or intermediate strains received nebulized ceftazidime and amikacin, whereas 17 patients infected with susceptible strains only received intravenous ceftazidime and amikacin. After 8 days, the curative rate was similar between groups, yet only in the intravenous group did antibiotic resistance develop. In a later study, Niederman et al. [16] studied 69 mechanically ventilated patients with Gram-negative VAP receiving nebulized amikacin with systemic antibiotics. They found that amikacin distributed well throughout the lung parenchyma, with very high tracheal and alveolar levels, while maintaining intravenous concentrations below the renal toxicity threshold. Those patients who received amikacin required significantly less systemic antibiotics than those who were given placebo. A recent meta-analysis [17] and a consensus guideline [18] took a closer look into the use of nebulized antibiotics. In particular, in the meta-analysis, Solé-Lleonart and collaborators [17] evaluated the effects of nebulized aminoglycosides or colistin, harnessed as adjunctive or substitutive therapies. The authors identified 11 studies, six of which were randomized trials, which were biased by methodological heterogeneity and limitations. The authors found that nebulized antibiotics in VAP patients reduced emergence of MDR, and clinical resolution was more frequent, specifically in VAP cases caused by resistant pathogens. Yet nebulized antibiotics increased the rate of respiratory complications, in particular hypoxemia after the nebulization, obstruction of the expiratory filter, and increase in peak airway pressure. In the consensus guideline by Rello and collaborators [18] this meta-analysis was acknowledged and the global use of nebulized antibiotics in units challenged by highly resistant pathogens. Ultimately, they advised against the use of nebulized antibiotics in VAP, primarily owing to the marginal scientific evidence.

\section{Recent disappointing results}

The randomized trial of amikacin/fosfomycin inhalation system for the adjunctive therapy of Gram-negative ventilator associated pneumonia (IASIS Trial) [1] recently evaluated nebulized amikacin/fosfomycin as an adjunctive therapy for the treatment of Gram-negative bacterial VAP. A similar study of the inhaled amikacin solution (BAY 416551) as adjunctive therapy in the treatment of Gramnegative pneumonia (INHALE I and II program) has recently been completed (ClinicalTrials.gov identifiers NCT 0179993 and NCT00805168). Turning first to the trial by Kollef and collaborators [1], this was a phase 2, multicenter, double-blind trial. More than 140 patients with VAP received intravenous meropenem or imipenem, and either a combination of nebulized antibiotics (300 mg amikacin and $120 \mathrm{mg}$ fosfomycin), twice daily for up to 10 days, or nebulized placebo. This study was preceded by various phase I studies [19-21], confirming that the combination of antibiotics, delivered through the PARI eflow system (PARI $\mathrm{GmbH}$, Germany), achieved very high tracheal aspirate concentrations and low systemic absorption. The primary outcome was variation in the clinical pulmonary infection score (CPIS) [22] - adjusted per baseline values-during the planned 10-day treatment period. Remarkable tracheal secretion concentrations of amikacin and fosfomycin were achieved during nebulization, higher than 7000 and 2000 $\mathrm{mg} / \mathrm{mL}$, respectively. Tracheal secretion colonization by Gram-negative pathogens also decreased in the treatment group, yet the study failed to demonstrate any benefit on CPIS variations, clinical cure rates, ventilator-and ICU-free days, and mortality. As for the recently completed INHALE program, 725 patients with Gram-negative VAP were randomized to receive standard intravenous therapy and either $400 \mathrm{mg}$ of nebulized amikacin every $12 \mathrm{~h}$ for 10 days or nebulized placebo. In this study, amikacin was nebulized through an inhalation system synchronized with the inspiratory phase, as detailed in previous publications [23, 24]. Unfortunately, on November 24th 2017, the main study promoter announced that inhaled amikacin did not demonstrate superiority over standard of care and nebulized placebo in the main endpoint [2]. Moreover, secondary outcomes did not favor the use of inhaled amikacin, including pneumonia-related mortality, early clinical response, number of days on mechanical ventilation, and number of ICU days. 


\section{Reappraisal of the latest clinical results and the next research chapter}

The results of the aforementioned latest studies are quite discouraging, particularly at the moment, when only limited efforts are being made to develop novel antibiotics. Nevertheless, we believe that many factors could have contributed to this lack of positive outcomes and in the following paragraphs we will provide our point of view.

On the one hand, the delivery of nebulized antibiotics into the distal portion of highly infected pulmonary regions filled with respiratory secretions seems just impractical (Fig. 1), irrespective of the best currently available nebulizers. Recent reports [25] have emphasized that even in healthy patients, only a limited amount of the nebulized dose is delivered into the lungs, predominantly in the proximal regions. Irrespective of the challenges encountered, several reports in VAP patients demonstrated very high concentrations in the epithelial lining fluid (ELF) [23] and tracheal aspirates [16], using the device described in the INHALE program.

We would like to point out several factors that may have been underestimated in aforementioned trials but which play a critical role in lung deposition of nebulized antibiotics during mechanical ventilation. Firstly, the extension and severity of lung infections critically affect lung distribution of nebulized antibiotics. Indeed, sufficient airway patency and alveolar opening are required to deposit antibiotics on the affected areas. In an interesting study [26] in pigs with pneumonia, lung tissue concentrations of nebulized antibiotics were significantly higher in pulmonary segments with early stages of lung infection than in segments with confluent pneumonia and lung abscess. The IASIS and INHALE II trials often started nebulization days after clinical diagnosis. Considering that VAP diagnosis is challenging and time-consuming, it is conceivable that many patients already presented consolidated pulmonary regions upon the first nebulization.

Secondly, both studies used vibrating mesh nebulizers. It is estimated that these devices increase the efficiency of aerosol delivery to $40-60 \%$ [27, 28]. However, there seem to be incongruities between the achieved antibiotic concentrations [16, 21, 24], the bactericidal effects corroborated by animal studies $[15,29]$, and the lack of clear benefits in phase III trials. In respect of the animal studies, one of the main limitations is that often the models do not fully resemble the complexity of patients admitted into an ICU and who develop VAP after a few days of intubation. For instance, animal studies are conducted in healthy young pigs without chronic pulmonary diseases. In addition, nebulization of antibiotics in these pre-clinical experiments initiates immediately upon development of pneumonia, which is difficult to reproduce in clinical settings.

Thirdly, it is well acknowledged that the mass median aerodynamic diameter (MMAD) should range between 1 and $5 \mu \mathrm{m}$ to reach distal airways and alveoli [30, 31]. Humidification affects lung deposition of nebulized antibiotics $[30,31]$, because controlled in vitro studies demonstrated that conditioning inspiratory gases lead to an increase in

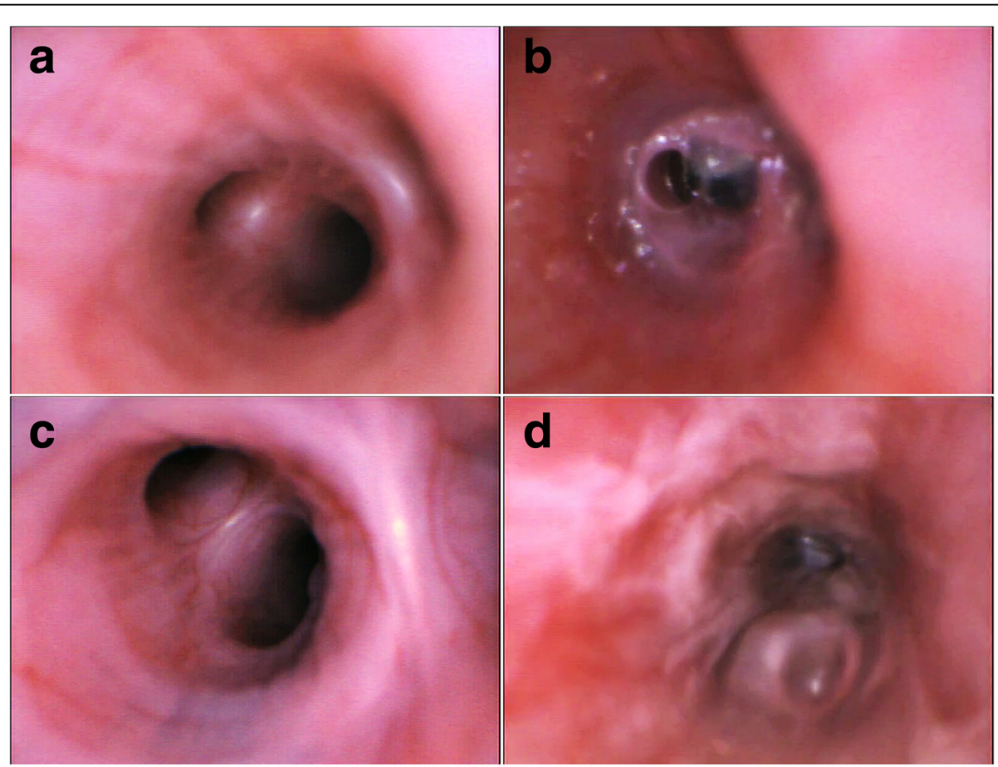

Fig. 1 Bronchoscopic evaluation of mechanically ventilated Large White Landrace pigs challenged by Pseudomonas aeruginosa. a Main right upper bronchus, prior to bacterial challenge; of note no abnormalities can be found. $\mathbf{b}$ After $24 \mathrm{~h}$ from inoculation of $15 \mathrm{~mL}$ of $10^{7}$ colony forming units of $P$. aeruginosa, the distal portion of the right middle bronchus is copiously filled with purulent secretions with a reduction of the distal bronchi by more than $60 \%$. c Main right medium bronchus, prior to bacterial challenge, with no abnormalities. d After $24 \mathrm{~h}$ from inoculation of $15 \mathrm{~mL}$ of $10^{7}$ colony forming units of $P$. aeruginosa, the bronchial mucosa is highly hyperemic and retained purulent secretions are evident throughout the bronchus, almost completely obstructing distal bronchi 
MMAD [32] and, potentially, to deposition on endotracheal tube and ventilator circuits [33]. In the IASIS and INHALE II trials, humidification was maintained throughout nebulization, so as to slightly increase the MMAD. Nonetheless, considering that ICU patients with pneumonia have heterogeneous requirements with regard to the level of ventilatory assistance, and the performance of humidification systems may vary [34, 35], any inference derived from highly controlled in vitro environments might be difficult to reproduce in clinical settings. Following the same line of thought, airflow turbulence should be avoided during nebulization [36]. Some investigators suggest that ventilatory parameters should be adjusted, i.e., using volume-control mode, constant inspiratory flow [37], and low minute ventilation and respiratory frequency. Furthermore, an inspiratory-toexpiratory ratio $\leq 50 \%$ and an end-inspiratory pause representing $20 \%$ of the duty cycle should be used to provide enough time for aerosol sedimentation in the alveolar space [38]. These recommendations are difficult to apply in busy, understaffed units, and which may lack expertise in respiratory management. Therefore, in the latest clinical trials, investigators prioritized straightforward administration of nebulized antibiotics, avoiding challenging ventilatory adjustments. This may have resulted in an unpredictable delivery of the dosed antibiotic, specifically in patients requiring high minute ventilation, on pressure-control ventilation, or ventilated with shorter inspiratory time.

Finally, we would like to raise our concerns regarding the potential methodological biases in the design of these latest clinical studies. The IASIS and INHALE II trials were intended to demonstrate superiority in clinical outcomes such as change in CPIS or mortality. These outcomes were fully endorsed by the American Food and Drug Administration agency, though from a research and clinical standpoint, the reliability of these parameters is questionable. For instance, the CPIS was originally designed to diagnose VAP [22], rather than to evaluate the response to treatment, whereas survival is an outcome not closely related to VAP, difficult to achieve in severely critically ill patients, and can also be biased due to variations in standards of treatment between countries or clinical departments. In addition, many experts in this field would argue that the use of nebulized antibiotics could have merits in patients with difficult-to-treat infections, e.g., caused by MDR, extensively drug-resistant (XDR) or even PDR pathogens. Theoretically, the inclusion of a large proportion of these patients would certainly have helped in detecting positive outcomes and would have highlighted the most appropriate clinical indication of this therapeutic strategy.

Considering the above arguments, we would like to present a few thought-provoking ideas to point toward new directions for proficient research. Turning first to the pre-clinical trials, it would be ideal to conduct comprehensive studies in larger animals before designing clinical studies. In particular, animal studies could elucidate key microbiology and pharmacology factors impossible to obtain in ICU patients, by means of postmortem analysis of pulmonary tissue [39]. Moreover, lung deposition could be accurately measured in these models through inhalation of radio-labeled tracers tracked by gamma scintigraphy or positron emission tomography [40].

Secondly, although investigators have tried to achieve the highest pulmonary concentrations of nebulized antibiotics, we call attention to the risks of pulmonary injury associated with these drugs, specifically in lungs already damaged by a primary infectious-related insult. With this in mind, future animal studies should also evaluate the pulmonary effects of these antibiotics, while at the same time achieving the highest concentrations.

Thirdly, considering pathogenesis of VAP and the potential for mucus retention and alveolar collapse, future investigations should look into the optimal timing to initiate nebulization of antibiotics. Likewise, in future randomized trials, earlier treatments should be given preference.

Fourthly, based on the latest negative findings, the longstanding optimistic view that ventilatory settings should not be adjusted to optimize delivery of nebulized antibiotics seems implausible, and should be reappraised in future studies. Additionally, most of the evidence on the effects of ventilatory settings and humidification on deposition of nebulized antibiotics seems outdated, relying on studies that used earlier generations of nebulizers. Consequently, larger efforts should be made to validate performance of novel technology, testing a large variety of settings in reliable models of critically ill ventilated patients.

Finally, we are firmly convinced that nebulized antibiotics should be primarily applied against pathogens virtually untreatable through standard intravenous treatment. To this end, patients with MDR, XDR, and PDR pulmonary infections should be the primary focus in future studies. Additionally, given the promising results [12] of the effects of nebulized antibiotics as a preventive strategy for drug resistance, we are inclined to believe that it would be reasonable, in the era of MDR, to redirect the goals of this promising therapy toward the prevention of selective pressure and avoidance of MDR development.

\section{Conclusions}

Irrespective of the most recent unsuccessful findings, we believe that nebulized amikacin for severe pulmonary infections still offers promising prospects in the ICU. Nevertheless, several methodological and technical challenges need to be overcome before embracing this therapeutic strategy. We call for a substantial body of future basic and clinical research to further investigate the principles of pulmonary nebulization during mechanical ventilation and to validate efficacy and safety of the most 
innovative nebulizers, and, last but not least, we call for clinical studies testing feasible and reliable outcomes to ultimately decide whether nebulized amikacin should be applied-and to whom.

\section{Abbreviations}

CPIS: Clinical pulmonary infection score; ELF: Epithelial lining fluid HAP: Hospital-acquired pneumonia; ICU: Intensive care unit; MDR: Multi-drugresistant; MMAD: Median aerodynamic diameter; PDR: pan-drug resistant; VAP: Ventilator-associated pneumonia; XDR: extensively drug-resistant

\section{Acknowledgements}

We deeply thank Andrea Meli, MD, University of Milan, for his valuable help with Fig. 1.

\section{Funding}

Not applicable.

\section{Availability of data and materials}

Not applicable.

\section{Authors' contributions}

AT and GLB wrote the ms. AM and DB have assisted in ms preparation and participated in animal studies regarding inhaled antibiotics. All authors read and approved the final manuscript.

\section{Ethics approval and consent to participate}

Not applicable.

\section{Consent for publication}

Not applicable.

\section{Competing interests}

Gianluigi Li Bassi and Antoni Torres have received, through their affiliated institution, research funds from Bayer HealthCare Pharmaceuticals LLC (Germany), the manufacturer of NKTR-061 and BAY41-6551, and Cardeas (USA), the manufacturer of the combination amikacin-fosfomycin inhaled solution. None of the remaining authors have any potential conflict of interest related to the main topic of this article.

\section{Publisher's Note}

Springer Nature remains neutral with regard to jurisdictional claims in published maps and institutional affiliations.

\section{Author details}

'Department of Pulmonary and Critical Care Medicine, Hospital Clinic, Calle Villarroel 170, Barcelona 08036, Spain. ${ }^{2}$ Institut d'Investigacions Biomèdiques August Pi i Sunyer (IDIBAPS), Barcelona, Spain. ${ }^{3}$ Centro de Investigación Biomedica En Red- Enfermedades Respiratorias (CIBERES), Barcelona, Spain. ${ }^{4}$ University of Barcelona, Barcelona, Spain. ${ }^{5}$ University of Genoa, Genoa, Italy.

Received: 13 December 2017 Accepted: 16 January 2018

Published online: 17 December 2018

\section{References}

1. Kollef MH, Ricard JD, Roux D, Francois B, Ischaki E, Rozgonyi Z, et al. A randomized trial of the amikacin fosfomycin inhalation system for the adjunctive therapy of Gram-negative ventilator-associated pneumonia: IASIS Trial. Chest. 2016;151:1239-46.

2. Bayer Ltd. http://press.bayer.com/baynews/baynews.nsf/id/Phase-IIIstudy-program-Amikacin-Inhale-addition-standard-intubatedmechanically-ventilated-patients?OpenDocument\&sessionID= 1512472033. Accessed 24 Nov 2017

3. Kalil AC, Metersky ML, Klompas M, Muscedere J, Sweeney DA, Palmer LB, et al. Management of adults with hospital-acquired and ventilator-associated pneumonia: 2016 Clinical practice guidelines by the Infectious Diseases Society of America and the American Thoracic Society. Clin Infect Dis. 2016; 63:e61-e111.

4. Warren DK, Shukla SJ, Olsen MA, Kollef MH, Hollenbeak CS, Cox MJ, et al. Outcome and attributable cost of ventilator-associated pneumonia among intensive care unit patients in a suburban medical center. Crit Care Med. 2003:31:1312-7.

5. Kollef MH, Hamilton CW, Ernst FR. Economic impact of ventilator-associated pneumonia in a large matched cohort. Infect Control Hosp Epidemiol. 2012; 33:250-6.

6. Martin-Loeches I, Torres A, Rinaudo M, Terraneo S, de Rosa F, Ramirez P, et al. Resistance patterns and outcomes in intensive care unit (ICU)-acquired pneumonia. Validation of European Centre for Disease Prevention and Control (ECDC) and the Centers for Disease Control and Prevention (CDC) classification of multidrug resistant organisms. J Inf Secur. 2014;70:213-22.

7. Torres A, Niederman MS, Chastre J, Ewig S, Fernandez-Vandellos P, Hanberger $\mathrm{H}$, et al. International ERS/ESICM/ESCMID/ALAT guidelines for the management of hospital-acquired pneumonia and ventilator-associated pneumonia: guidelines for the management of hospital-acquired pneumonia (HAP)/ventilator-associated pneumonia (VAP) of the European Respiratory Society (ERS), European Society of Intensive Care Medicine (ESICM), European Society of Clinical Microbiology and Infectious Diseases (ESCMID) and Asociación Latinoamericana del Tórax (ALAT). Eur Respir J. 2017:50:1-26.

8. Roberts JA, Lipman J. Pharmacokinetic issues for antibiotics in the critically ill patient. Crit Care Med. 2009:37:840-51.

9. Vandecandelaere I, Coenye T. Microbial composition and antibiotic resistance of biofilms recovered from endotracheal tubes of mechanically ventilated patients. Adv Exp Med Biol. 2015;830:137-55.

10. Smith BS, Yogaratnam D, Levasseur-Franklin KE, Forni A, Fong J. Introduction to drug pharmacokinetics in the critically ill patient. Chest. 2012;141:1327-36

11. Palmer LB. Aerosolized antibiotics in the intensive care unit. Clin Chest Med. 2011;32:559-74

12. Palmer LB, Smaldone GC. Reduction of bacterial resistance with inhaled antibiotics in the intensive care unit. Am J Respir Crit Care Med. 2014;189: 1225-33.

13. Trapnell BC, McColley SA, Kissner DG, Rolfe MW, Rosen JM, McKevitt M, et al Fosfomycin/tobramycin for inhalation in patients with cystic fibrosis with pseudomonas airway infection. Am J Respir Crit Care Med. 2012;185:171-8.

14. Goldstein I, Frederic W, Nicolas-Robin A, Ferrari F, Marquette $\mathrm{CH}$, Rouby J-J. Lung deposition and efficiency of nebulized amikacin during Escherichia coli pneumonia in ventilated piglets. Am J Respir Crit Care Med. 2002;166:1375-81.

15. Lu Q, Yang J, Liu Z, Gutierrez C, Aymard G, Rouby JJ, et al. Nebulized ceftazidime and amikacin in ventilator-associated pneumonia caused by Pseudomonas aeruginosa. Am J Respir Crit Care Med. 2011;184:106-15.

16. Niederman MS, Chastre J, Corkery K, Fink JB, Luyt CE, García MS. BAY41-6551 achieves bactericidal tracheal aspirate amikacin concentrations in mechanically ventilated patients with Gram-negative pneumonia. Intensive Care Med. 2012:38:263-71.

17. Solé-Lleonart C, Rouby JJ, Blot S, Poulakou G, Chastre J, Palmer LB, et al. Nebulization of anti-infective agents in invasively mechanically ventilated adults: a systematic review and meta-analysis. Anesthesiology. 2017;126: 890-908.

18. Rello J, Solé-Lleonart C, Rouby JJ, Chastre J, Blot S, Poulakou G, et al. Use of nebulized antimicrobials for the treatment of respiratory infections in invasively mechanically ventilated adults: a position paper from the European Society of Clinical Microbiology and Infectious Diseases. Clin Microbiol Infect. 2017;23:629-39.

19. Montgomery AB, Rhomberg PR, Abuan T, Walters KA, Flamm RK. Potentiation effects of amikacin and fosfomycin against selected amikacinnonsusceptible Gram-negative respiratory tract pathogens. Antimicrob Agents Chemother. 2014;58:3714-9.

20. Montgomery AB, Rhomberg PR, Abuan T, Walters KA, Flamm RK. Amikacinfosfomycin at a five-to-two ratio: characterization of mutation rates in microbial strains causing ventilator-associated pneumonia and interactions with commonly used antibiotics. Antimicrob Agents Chemother. 2014;58:3708-13.

21. Montgomery AB, Vallance $S$, Abuan $T$, Tservistas M, Davies A. A randomized double-blind placebo-controlled dose-escalation phase 1 study of aerosolized amikacin and fosfomycin delivered via the PARI Investigational eFlow $\left.{ }^{\circledR}\right)$ Inline Nebulizer System in mechanically ventilated patients. J Aerosol Med Pulm Drug Deliv. 2014;27:441.

22. Pugin J, Auckenthaler R, Mili N, Janssens JP, Lew PD, Suter PM. Diagnosis of ventilator-associated pneumonia by bacteriologic analysis of bronchoscopic and nonbronchoscopic "blind" bronchoalveolar lavage fluid. Am Rev Respir Dis. 1991:143:1121-9. 
23. Luyt CE, Clavel M, Guntupalli K, Johannigman J, Kennedy JI, Wood C, et al Pharmacokinetics and lung delivery of PDDS-aerosolized amikacin (NKTR061) in intubated and mechanically ventilated patients with nosocomial pneumonia. Crit Care. 2009;13:R200

24. Luyt CE, Eldon MA, Stass H, Gribben D, Corkery K, Chastre J. Pharmacokinetics and tolerability of amikacin administered as BAY41-6551 aerosol in mechanically ventilated patients with gram-negative pneumonia and acute renal failure. J Aerosol Med Pulm Drug Deliv. 2011;24:183-90.

25. Dugernier J, Reychler G, Wittebole X, Roeseler J, Depoortere V, Sottiaux T, et al. Aerosol delivery with two ventilation modes during mechanical ventilation: a randomized study. Ann Intensive Care. 2016;6:73.

26. Elman M, Goldstein I, Marquette CH, Wallet F, Lenaour G, Rouby JJ, et al. Influence of lung aeration on pulmonary concentrations of nebulized and intravenous amikacin in ventilated piglets with severe bronchopneumonia. Anesthesiology. 2002;97:199-206.

27. Ferrari F, Lu Q, Girardi C, Petitjean O, Marquette CH, Wallet F, et al. Nebulized ceftazidime in experimental pneumonia caused by partially resistant Pseudomonas aeruginosa. Intensive Care Med. 2009;35:1792-800.

28. Lu Q, Girardi C, Zhang M, Bouhemad B, Louchahi K, Petitjean O, et al. Nebulized and intravenous colistin in experimental pneumonia caused by Pseudomonas aeruginosa. Intensive Care Med. 2010;36:1147-55.

29. Tonnellier M, Ferrari F, Goldstein I, Sartorius A, Marquette CH, Rouby JJ. Intravenous versus nebulized ceftazidime in ventilated piglets with and without experimental bronchopneumonia: comparative effects of helium and nitrogen. Anesthesiology. 2005;102:995-1000.

30. Brain JD, Valberg PA. Deposition of aerosol in the respiratory tract. Am Rev Respir Dis. 1979;120:1325-73.

31. Miller DD, Amin MM, Palmer LB, Shah AR, Smaldone GC. Aerosol delivery and modern mechanical ventilation: in vitro/in vivo evaluation. Am J Respir Crit Care Med. 2003;168:1205-9.

32. Ferron GA, Kerrebijn KF, Weber J. Properties of aerosols produced with three nebulizers. Am Rev Respir Dis. 1976;114:899-908.

33. Dhand R, Guntur VP. How best to deliver aerosol medications to mechanically ventilated patients. Clin Chest Med. 2008;29:277-96.

34. Martin C, Papazian L, Perrin G, Bantz P, Gouin F. Performance evaluation of three vaporizing humidifiers and two heat and moisture exchangers in patients with minute ventilation > $10 \mathrm{~L} /$ min. Chest. 1992:102:1347-50.

35. Martin C, Thomachot L, Quinio B, Viviand X, Albanese J. Comparing two heat and moisture exchangers with one vaporizing humidifier in patients with minute ventilation greater than $10 \mathrm{~L} / \mathrm{min}$. Chest. 1995;107:1411-5.

36. Dolovich MA. Influence of inspiratory flow rate, particle size, and airway caliber on aerosolized drug delivery to the lung. Respir Care. 2000;45:597-608.

37. Dhand R. Maximizing aerosol delivery during mechanical ventilation: go with the flow and go slow. Intensive Care Med. 2003;29:1041-2.

38. Fink JB, Dhand R, Grychowski J, Fahey PJ, Tobin MJ. Reconciling in vitro and in vivo measurements of aerosol delivery from a metered-dose inhaler during mechanical ventilation and defining efficiency-enhancing factors. Am J Respir Crit Care Med. 1999;159:63-8.

39. Sibila O, Agustí C, Torres A, Baquero S, Gando S, Patrón JR, et al. Experimental Pseudomonas aeruginosa pneumonia: evaluation of the associated inflammatory response. Eur Respir J. 2007;30:1167-72.

40. Wenzler E, Fraidenburg DR, Scardina T, Danziger LH. Inhaled antibiotics for Gram-negative respiratory infections. Clin Microbiol Rev. 2016;29:581-632. 